\title{
Neutrino signals by Upward Tau airshowering at Earth horizons and by Muon airshowering at Moon shadows
}

D. Fargion, ${ }^{a, b, *}$ P.G. De Sanctis Lucentini ${ }^{c}$ and M. Y. Khlopov ${ }^{d, e, f}$

${ }^{a}$ Physics Department Rome University 1, P.le A. Moro 5, 00185, Rome, Italy

${ }^{b}$ INFN Rome1, P.le A. Moro 2, 00185, Rome, Italy

${ }^{c}$ Physics Department, National University of Oil and Gas Gubkin University

65 Leninsky Prospekt, Moscow, 119991, Russia

'Centre for Cosmoparticle Physics "Cosmion"

National Research Nuclear University ”MEPHI”, Kashirskoe Sh. 31, Moscow 115409, Russia

${ }^{e}$ Université de Paris, CNRS, Astroparticule et Cosmologie, F-75013 Paris, France

${ }^{f}$ Institute of Physics, Southern Federal University, 344090, Rostov on Don, Russia

E-mail: daniele.fargion@fondazione.uniroma1.it

\footnotetext{
${ }^{*}$ Presenter
} 
Neutrinos are invisible, but their interactions with matter and their leptons signature leave an observable trace. Due to the huge atmospheric neutrino noise produced by cosmic ray rain present detectors are hidden deep in underground, as SK or IceCube detectors. At highest energy, atmospheric signal are found to be overcome by a new flavor signature. Because of it at those edges there is much hope for revealing highest energy neutrino as an astronomy, well above tens $\mathrm{TeVs}$.

We remind that the three neutrinos and antineutrinos flavors show different roles. Traces of GeVs-TeVs electrons radiate a lot, so they are short (on the order of meters) within solid matter. Muons, even if unstable, they radiate much less, so they are much more long life and penetrating (range of kilometers at TeVs eneregy in matter). These muons may be born inside a rock and escape from matter, as skimming from mountains; they may also decay over great distances, much larger than the size of Earth. In analogy, taus, the third and most unstable leptons, are hardly formed from cosmic rays secondaries. Therefore they arise mainly from astrophysical neutrinos, that are democratically mixed during stellar and cosmic oscillating flights. Tau are also the most penetrating in principle but because they are extremely unstable, they are relevant only at PeVs energy edges. They may rise as a inner cascade and a later decay as a larger shower in icy: the so called double bang.

The tau penetrability range and decay in rock, at PeV energy, is around 49 meters; the $\tau$, once escape in air, may decay in air-shower. For instance their escaping from a mountain or from the Earth, is amplified in a widest area and by richest secondaries as rare upgoing airshowers. Therefore, for more than twenty years they have been advocated and proposed as a new filtered neutrinos astronomy no longer hidden in underground detectors, free from most atmospheric noises. These are the signals searched by present ongoing experiments from mountains, valleys and from space: the tau airshowers.

Nevertheless also muons at TeVs or higher energy can in principle decay in flight, but mainly only from the Moon distances. Their secondary electron may trace wide airshower on Earth atmosphere as gamma ones. Because the terrestrial and nearby magnetic fields, the bending begin to fade above $6.4 \mathrm{TeV}$; just above $61.2 \mathrm{TeVs}$ the muons are mostly no longer able to decay in flight.

Therefore, rarest gamma-like airshower in largest LHAASO like array, in the $6.4-61.2 \mathrm{TeV}$ windows, may be discovered in future years. The widest thousands of kilometer-size gamma array such as GRAND ARRAY can detect these trough going muons escaping from the Moon, decaying as electron or gamma on terrestrial airshowers.

More energetic and fragmented decays can also rarely occur, from PeVs tau from the Moon. The corresponding solar shadow is opaque to neutrinos. But their skimming PeVs CRs could still shine more likely muons, whose decay in flight may also soon rise as a gamma corona halo around our Sun, with a possibly already detectable signal in LHAASO.

$37^{\text {th }}$ International Cosmic Ray Conference (ICRC 2021)

July 12th-23rd, 2021

Online - Berlin, Germany 


\section{Introduction}

Neutrinos are weakly interacting particles observable by their interaction with matter and by their lepton track or showers. Due to the weaknesses of the interaction, a huge detector mass, such as the IceCube one, is required. Usually a $v$ neutrino detector should be screened from overabundant downward penetrating noises as the muons, the penetrating downward cosmic ray secondaries. Therefore,most detectors are hidden in deep underground as Icecube (one km cubic ice in South Pole). As we shall underline in present article, there are novel ways to discover UHE, (Ultra High Energy) TeVs-PeVs-EeVs neutrinos: they are based mainly on tau airshowers in deep valley, [4], at Earth edges [2], [5] and rarely by muon decay in flight from the Moon.[11]. Let us remind that Cosmic Rays (CR) secondaries are also made by $v$ ones, the polluting atmospheric neutrino noises. IceCube filter most of the huge $\mathrm{CR}$ muons raining downward noises selecting mainly upcoming ones made by UHE $v$, contained inside. Icecube also hope to disentangle astrophysical neutrinos above highest energies, because, they noticed a revolutionary flavor change: from a High Energy Starting Events (HESE) ruled by muon tracks at TeVs energy to a showers dominated one, above $30-60 \mathrm{TeV}$, ruled mainly by electrons, Neutral Current (NC) channels and, in principle, by taus neutrino interactions. Finally through-going upward muons near or above hundred TeV energy are used to enlarge the IceCube neutrino mass volume. Sponge like enlarged IceCube array may offer soon higher energetic muon tracks at sharp and highest rate: [10]. Our days neutrino astronomy is based on the IceCube muon tracks exceeding at tens TeV longest distances as shown in Fig.1.

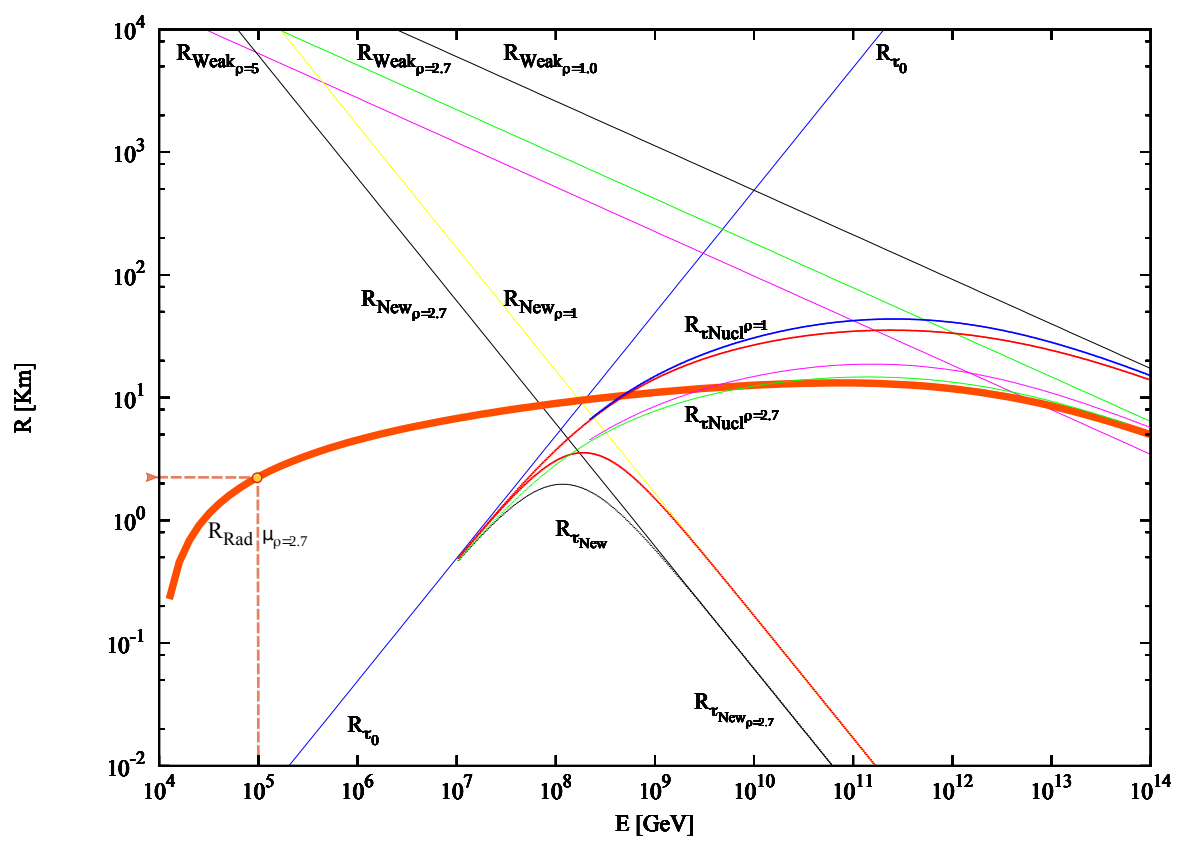

Figure 1: The range of leptons $\mu$ muon and $\tau$ at different energies inform us on their main penetrabilty and indirectly on their related probability to escape upward from any surface [5]. The $\mu$ muon tracks at $10^{13} \mathrm{eV}$ or $10^{14} \mathrm{eV}$ overcome a kilometer range while a corresponding tau only 0.49 or 4.9 meters. This makes nearly a thousand time more probable muons escape than tau ones from Moon crust. 
The consequence of the unstable tau lepton life, as shown in Fig.1, is the dominance of tau signals mainly above PeVs or better tens PeV, ideally overcoming muon ranges at GZK edge energy, $\mathrm{EeV}$ ones. Therefore,tau airshower might be revealed at PeVs inside deep and wide valley, where mountains are the neutrino calorimeter to let tau escape horizontally. The same Earth crust may act as a huge valley while being be observed from a mountain, a balloon or a satellite. See left side of Fig.2.

However the TeVs muon are too long life to be able to decay even inside the Earth radius distances. But TeVs muons may decay in flight from the Moon. The present results and bounds by AUGER and IceCube favor the hundred $\mathrm{TeV}$ neutrino energy for first astrophysical signals, with a possible cut-off above the PeVs energy. Therefore,the tens $\mathrm{TeV}$ neutrino energy windows is the most easy one to be revealed. At these $10^{13} \mathrm{eV}$ energy, the tau range track $L_{\tau}=0.49 \mathrm{~m}$ in too small to compete with the muon one in ice, near $L_{\mu}=1 \mathrm{~km}$. These makes muon escape from the Moon at ten $\mathrm{TeV}$ energy nearly a thousand times more probable than a tau one.

Moreover at these energy the Moon distance it is tuned to let any muon to decay in flight before reaching, as an electron, Earth atmosphere, where it is able to air-shower in a detectable way and in wide area. LHAASO square kilometer size might be able to observe such a gamma-like signal inside the Moon shadow disk. See right side of Fig.2.

\section{The Earth edges and the Moon's shadow as an ideal $v_{\tau}, v_{\mu}$ filter and screen}

The new Neutrino astronomy based on Tau Air-shower might rise mainly above a few or tens PeVs, because the shortest tau life time become rilevant only at such UHE energy. Above hundred PeVs tau range overcome even muon ones and become the best tool to discover GZK signals (the UHECR neutrino secondaries of cosmic $2.75 \mathrm{~K}$ opacity). Because of these extreme energies, the Earth opacity is relevant, forcing us to consider the upward or better horizontal upgoing tau airshower, skimming small Earth cords. Therefore,one needs huge detector areas (as GRAND or
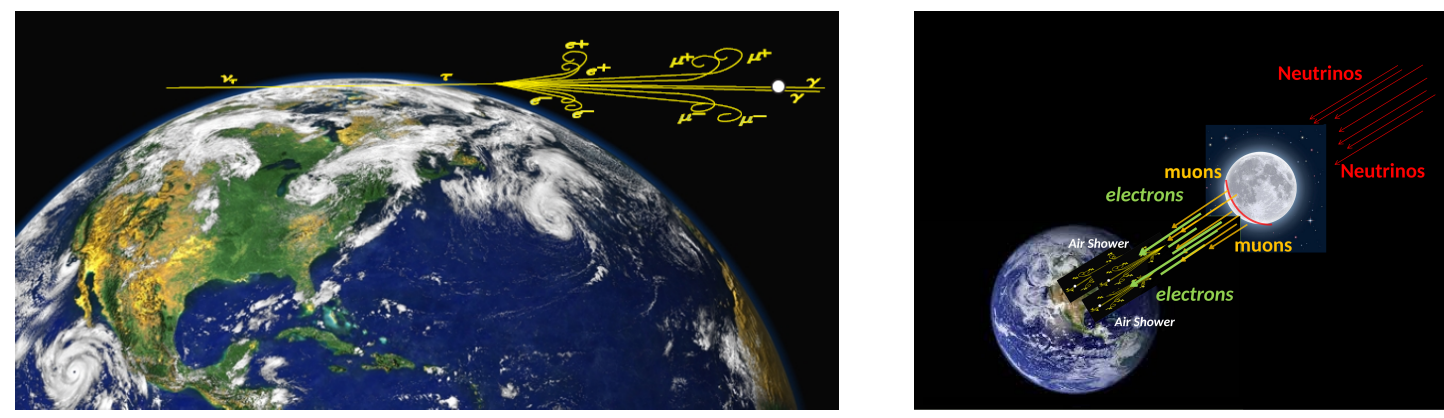

Figure 2: Left Any astrophysical ultra-relativistic $P e V s-E e V s v_{\tau}$ may interact on Earth crust, upward skimming the Earth: its born $\tau$ may decay in flight in terrestrial atmosphere leading to huge tau airshowers shining detectors in mountains, balloons or satellites. [6-8] Their spread nature require array detectors either in vertical and-or in horizontal structures, [3]. Right In analogy tens TeVs muons, made by $v_{\mu}$, may escape upward the Moon crust pointing toward the Earth, leading by muon beta decay in flight, a surprising electromagnetic (gamma-like) airshower flashes on Earth inside the Moon's shadow. This signal it is very rare, once every decade in LHAASO. Future enlarged GRAND array or even AUGER ones as well as POEMMA satellites might one day be able to discover such peculiar lunar neutrino shadows signals. 
AUGER array), possibly located at top mountains (as Magic, CTA or ASHRA) or even flying with balloons or satellites [1]. There is a key point often neglected by researchers: To disentangle tau airshower directionality at horizons (competitive with skimming hadron $\mathrm{CR}$ airshowers) one need an array (not just an unique or just a couple of detectors). The need of vertical zenith distinction imply a double or triple crown-like array, as a Pisa tower, at the mountain or balloons top edges [3]. Moreover highest altitude where tau PeVs-EeVs UHE airshower occur are so pressureless and diluted to allow the airshower to be easely opened and bent by geomagnetic fields into twin or triple very thin fan-like spiral shape [3], different than usual downward cone like tree vertical airshower. Because of it future Tau airshower array should be more and more projected as arrays: in equatorial latitude, because of azimuthal geomagnetic field, in vertical arrays. Along the North or south Poles, the array must be more spread in Horizontal crown.

\section{Moon's shadow transparence to $v_{\tau}, v_{\mu}$ Pevs energy}

There are questions about the quality of UHE neutrinos across the Moon. Are Moon neutrino polluted by atmospheric noise? The obvious answear is not: no atmosphere, no noise.

The next question on the ability for muon neutrino to cross the Moon diameter, reaching later on the Earth is related to the neutrino opacity across stellar, planet, Moon bodies. As shown in Fig.3 the Moon is transparent up ten $\mathrm{PeV}$ to neutrinos. This guarantee that tens $\mathrm{TeV}$ escaping muons made on Moon crust are not suppressed by any Moon opaciy. The tens $\mathrm{TeV}$ muons might cross anyway few kilometers along the Moon crust: the interaction probability, given in first approximation by the ratio, among the muon range over the neutrino interaction distance in rock material, is above

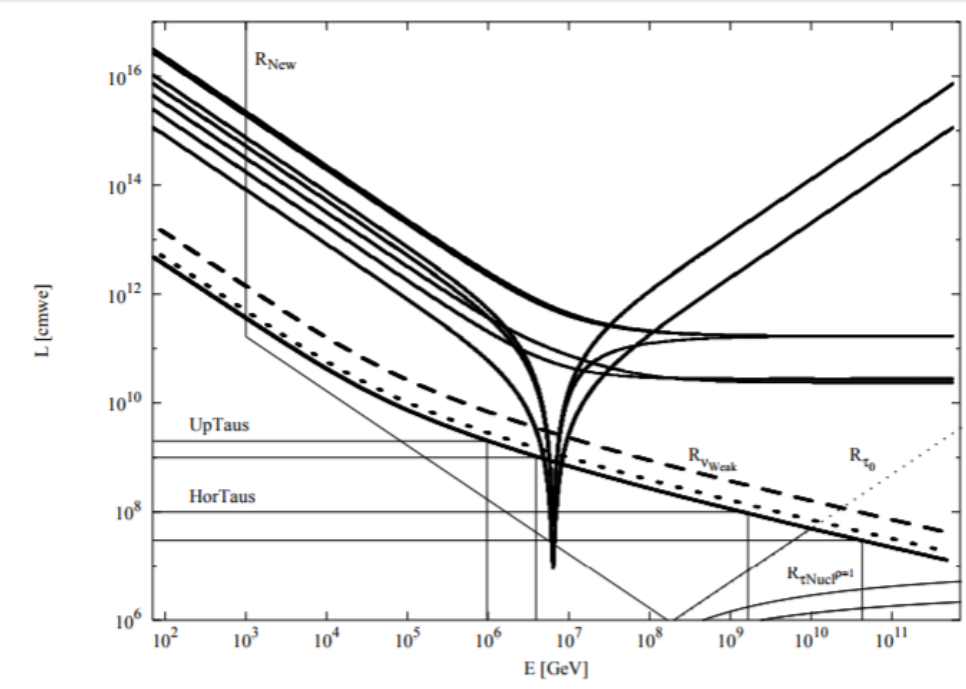

Figure 3: The opacity to neutrinos across the Sun is severe, below a few TeV[9, 12]. The Earth opacity to UHE neutrinos along its diameter begins above $30 \mathrm{TeV}$; the corresponding Moon opacity arise at several or ten PeVs. This imply that the Moon is not much suppresing hundreds or tens TeVs muon neutrinos upgoing toward the the Earth. Moon it is transparent at those energies. Their interaction in external lunar crust facing the Earth is possible; their muon escape probability at $30 \mathrm{TeVs}$ is near $3 \cdot 10^{-5}$ capable anyway to send a rare muon and its decay electron signal to the Earth. 
$3 \cdot 10^{-5}$ at $30 \mathrm{TeV}$ energy. The small solid angle of the Moon shadow and the paucity of the interaction probability makes the signal at Earth in LHAASO very rare, but in principle anyway detectable in future enlarged arrays.

\section{Undeflected muons inside the Moon Shadows}

Let's remind that the decay time of a muon is $\tau_{\mu}=2,197 \cdot 10^{-6} \mathrm{~s}$ and that the muon mass is $m_{\mu}=105,65 \mathrm{MeV}$, in order to estimate the distance life in flight of an ultra-relativistic $\mu^{ \pm}$: $L_{\mu} \simeq 6234,26 \mathrm{~km}\left(\frac{E_{\mu}}{1 \mathrm{TeV}}\right)$

There is to remind the extraterrestrial magnetic field bending to any $\mathrm{Z}$ nuclei charge (as an unique muon charge: $\mathrm{Z}=1) . \Delta \theta \simeq 1,6^{\circ}\left(\frac{E}{1 \mathrm{TeV}}\right) \cdot Z$

Any CR nuclei as well any proton or electron signal may pollute at given energy the dark Moon shadows. Incidentally this pollution of positive and negative protons allowed first bound on anti proton spectra since long time ago. Indeed the Moon radius angle size it is much smaller than the bending one above, as observed from Earth: $\Delta \theta_{\text {moon }}=\arctan \left(\frac{R_{\text {moon }}}{D_{\oplus-\text { moon }}}\right) \simeq 0,26^{\circ}$

Let us notice that the Moon solid angle with respect to the whole sky is just a tiny fraction: $\frac{\Delta \Omega_{\text {moon }}}{\Delta \Omega_{4 \pi}} \simeq 5,3 \cdot 10^{-6}$

There is, therefore, a narrow but important windows of space and energy where muons reach the Earth as electron but they are not much bent outside the Moon Shadows. This range is easely found from above as:

$$
6,4 \mathrm{TeV} \lesssim E_{\mu} \lesssim 61,66 \mathrm{TeV}
$$

Analogous arguments were extensively discussed in details for $v_{\tau}, \bar{v}_{\tau}$ and $\bar{v}_{e}$ produced inside the Earth crust and escaping as $\tau$-airshowers.

\section{Solar opacity to $v_{\tau}, v_{\mu} \mathbf{T e V}$ energy and the CR secondaries around the Sun corona}

The PeV CR may skimm the solar atmosphere toward us, leading to tens-hundred penetrating $\mathrm{TeV}$ muons (or few direct gamma and electrons secondaries) shining at Earth atmosphere. These events are only around one percent of the solar disk external disk and they might produce a tiny, (not negligible), skimming muons (and final electrons) shining in LHAASO external solar shadows. The paucity of gamma background (less tha $10^{-4}$ respect hadron $\mathrm{CR}$ ) at tens $\mathrm{TeV}$, makes this signal observable in LHAASO:

\subsection{Solar Corona atmosphere density profile allowing skimming $\mathrm{CR}$ and muons}

The atmosphere profile of solar corona may allow to define the ring around the most diluted atmosphere where UHECR (PeVs-EeVs) may shine skimming to Earth. See Fig.5. The shaded narrow external ring is marked showing the area where skimming CR may eject muons to Earth. These gamma-like signals might be observed by LHAASO in near years. 


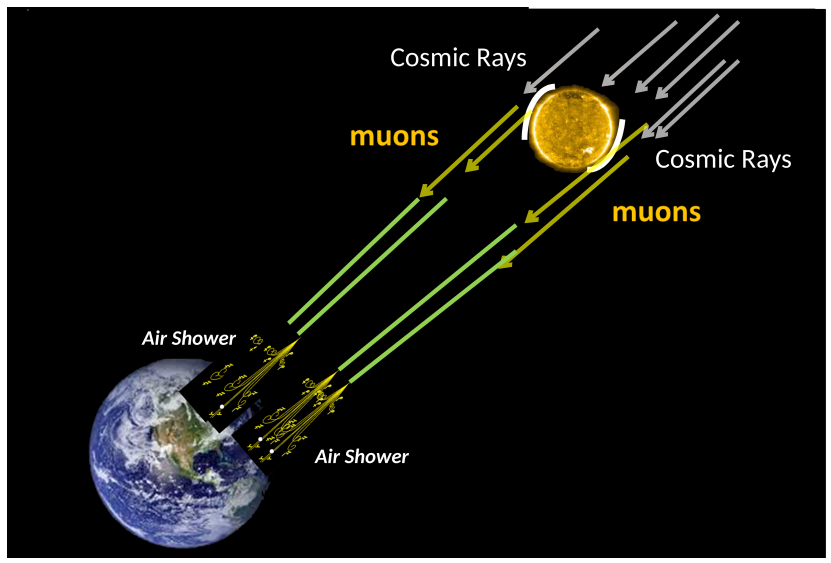

Figure 4: The peculiar skimming PeVs CR around the solar corona along the thinner external solar atmosphere may shine hundreds $\mathrm{TeV}$ s muons and the consequent (by beta decay in flight) electron signals in a ring surrounding the solar shadows. This CR hadronic component, is converting a tiny (nearly one percent) hadrons along the external corona in muons and final gamma-like signals hitting on Earth atmosphere. Hadron $\mathrm{CR}$ at Hundred TeVs or PeVs are ten thousand more abundant than gamma ones. Therefore,the rise of a thin ring gamma-like around at one percent ratio along the solar ring corona, is relevant and detectable. This signal indeed might soon be detectable within a year or a few by LHAASO gamma array.
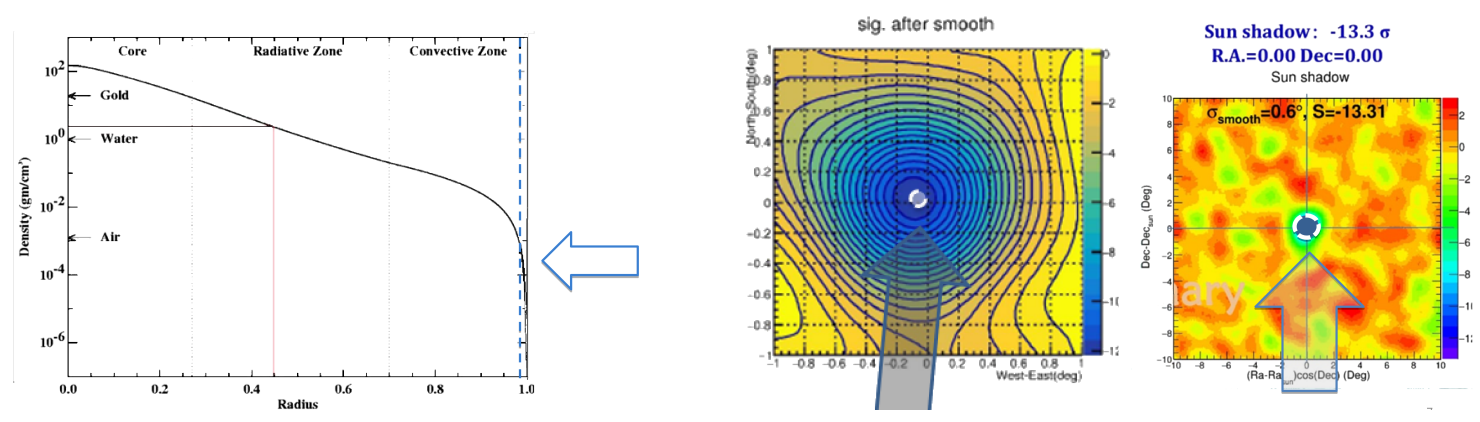

Figure 5: Left The external solar atmosphere density profile. The thin external ring is the place where the skimming PeVs CR are able to amplify a shower, to let their electron pairs, gamma secondaries and the harder muon bundle penetrate nearly unabsorbed, and to eject electrons and muon bundles toward the Earth, as it is marked by a vertical dashed line. The solar disk shadow would be therefore,enriched by the surprising gamma-like ring of air-showers whose presence must be soon discovered by LHAASO.

Center Moon shadow in signals [13]. A very schematic expected signature of hundred TeV muon traces, by UHE astrophysical neutrinos, shining as a very rare gamma-like shower inside the Moon shadows.

Right A much more probable and somehow just atmospheric CR skimming into a ring in an external gammalike signature. The rare Moon shadow flashes are Neutrino Astronomy signals; the Solar gamma ring is a more mundane, but much probable and detectable CR-muon-gamma trace in LHAASO. 


\section{Conclusions}

The presence of gamma-like TeVs signals inside the Moon shadow is possible observable in LHAASO or Grand in far (decades) future. A similar, more probable gamma ring around the solar corona is also possible in a very near future: a signal not connected to neutrinos, but related to the PeVs skimming CR and their muon secondaries which decay as electron in flight. These signals amplify the understanding of solar atmosphere fields and CR spectra. Once the solar gamma ring will be observed, the Moon shadows and its rarest gamma shining would be opening a whole new neutrino astronomy horizons.

\section{Dedication and Acknowledgements}

We dedicate this contribution to the memory of our most admired giant of Physics: Steven Weinberg, who with W. Pauli, M.Conversi, M. Peirl and B. Pontecorvo discovered the unified Electroweak model and the physics of the Neutrino on which our astrophysical studies are based; we also want to dedicate the article to Miguel Virasoro, whose earliest contributing to the string model opened the first horizons to a final unified theory. The work by M.K. has been performed with a support of the Ministry of Science and Higher Education of the Russian Federation, Project "Fundamental problems of cosmic rays and dark matter", No 0723-2020-0040.

\section{References}

[1] M. Bagheri, P. Bertone, I. Fontane, E. Gazda, E. G. Judd, J. F. Krizmanic, E. N. Kuznetsov, M. J. Miller, J. Nachtman, Y. Onel, A. Nepomuk Otte, P. J. Reardon, O. Romero Matamala, L. Wiencke, and f. t. J.-E. Collaboration. Overview of Cherenkov Telescope on-board EUSO-SPB2 for the Detection of Very-High-Energy Neutrinos. PoS, ICRC2021:1191, 2021.

[2] D. Fargion. Discovering Ultra High Energy Neutrinos by Horizontal and Upward tau Air-Showers: Evidences in Terrestrial Gamma Flashes? Astrophys. J., 570:909-925, 2002.

[3] D. Fargion. Vertical Array in Space for Horizontal Air-Showers. PoS, EPS-HEP2009:104, 2009.

[4] D. Fargion, A. Aiello, and R. Conversano. Horizontal tau air showers from mountains in deep valley: Traces of UHECR neutrino tau. 26th International Cosmic Ray Conference, pages 396-398.

[5] D. Fargion, P. G. De Sanctis Lucentini, and M. De Santis. Tau air showers from earth. Astrophys. J., 613:1285-1301, 2004.

[6] D. Fargion, M. De Santis, P. De Sanctis Lucentini, and M. Grossi. Muon and gamma bundles tracing up-going tau neutrino astronomy. Nuclear Physics B, 136:119, 2004.

[7] D. Fargion, M. Grossi, M. De Santis, and P. G. De Sanctis Lucentini. Rates of horizontal tau air-showers observable by satellites. In 35th COSPAR Scientific Assembly, 12005.

[8] D. Fargion, M. Grossi, M. De Santis, P. G. De Sanctis Lucentini, M. Iori, A. Sergi, and F. Moscato. Crown detectors to observe horizontal and upward air-showers. Adv. Space Res., 37:2132-2138, 2006.

[9] D. Fargion, M. Khlopov, R. Konoplich, P. De Sanctis Lucentini, M. De Santis, and B. Mele. Ultra high energy particle astronomy, neutrino masses and tau airshowers. RECENT RES.DEVEL.ASTROPHYS., 1:395, 32003.

[10] D. Fargion, P. G. D. S. Lucentini, and M. Khlopov. A shell like kilometer spaced array around icecube and a honey comb one. In Proceedings of 37th International Cosmic Ray Conference - PoS(ICRC2021). Sissa Medialab, July 2021.

[11] D. Fargion, P. Oliva, P. G. De Sanctis Lucentini, and M. Y. Khlopov. Signals of he atmospheric $\mu$ decay in flight around the sun's albedo versus astrophysical $v_{\mu}$ and $v_{\tau}$ traces in the moon shadow. International Journal of Modern Physics D, 27(06):1841002, 2018.

[12] D. Fargion and Others. Splitting neutrino masses and showering into sky. Nuclear Physics B - PS, 168:292-295, 2007.

[13] Y. Wang, Z. Cao, Z. Zeng, L. Ma, and Y. Nan. The Energy Scale Calibration using the Moon Shadow of LHAASO-WCDA Detector. PoS, ICRC2021:356, 2021. 